\title{
Determination of crystalline forms by solid-state NMR and electron diffraction
}

\author{
Yusuke Nishiyama $^{1}$ \\ ${ }^{1}$ RIKEN CLST-JEOL Collaboration Center, Yokohama, Japan \\ E-mail: yunishiy@jeol.co.jp
}

We demonstrate a combined approach to determine the crystalline polymorphs of small-organic micro-crystalline molecules, using solid-state NMR (sSNMR) and electron diffraction (ED) [1]. Powder X-ray diffraction is a widely used method for crystalline form determination, however, it sometimes fails in mixture samples because of severe overlaps of diffraction peaks. For mixture samples, $13 \mathrm{C}$ cross-polarization magic-angle spinning (CPMAS) is power full tool because of the sensitivity of $13 \mathrm{C}$ chemical shifts to the molecular conformation and high resolution of peaks. However, crystalline polymorphs with very similar conformations sometimes give identical 13C CPMAS spectra, failing to distinguish crystalline form. ED can in principal be very useful method to distinguish crystalline form even for mixture samples because ED requires only micro- or even nano-crystalline sample due to the strong scattering power, enabling measurement of single crystal ED pattern from powder mixture samples. However, its application is usually limited only to inorganic molecule. This is because the ED measurements of organic molecules are very challenging due to the sample degradation by electron irradiation. We overcome these difficulties by $1 \mathrm{H}$ double-quantum (DQ) / single-quantum (SQ) correlation experiments at very fast MAS together with ED observation with a mild electron irradiation using highly sensitive CMOS camera. Since $1 \mathrm{H}$ is located at the surface of the molecule, $1 \mathrm{H}$ chemical shift is sensitive to not only molecular conformation but also molecular packing. In addition, intermolecular DQ/SQ correlation is very sensitive method to distinguish molecular packing. This achieves alternative way to crystalline form determination from the difference in molecular packing. The very fast MAS also allows to measure $1 \mathrm{H} / 14 \mathrm{~N}$ correlation spectra which clearly distinguish the protonated state. On the other hand, ED gives very different patterns for each crystalline polymorph. The experiments were demonstrated on L-histidine samples in Lhistidine. $\mathrm{HCl} . \mathrm{H} 2 \mathrm{O}$, orthorhombic L-histidine, and monoclinic L-histidine.

[1] T. Oikawa, M. Okumura, T. Kimura and Y. Nishiyama, Acta Cryst. C73 (2017) 219-228.

Keywords: solid-state NMR, electron diffraction, crystalline polymorphs 